\title{
Virtual Reality: Principles and Applications
}

\author{
Frédéric Merienne \\ Le2i, Arts et Metiers, France
}

\begin{abstract}
Virtual reality aims at immersing a user in a virtual environment. Dedicated virtual reality technologies of human-computer interaction enable to make the link between the user and a virtual environment in capturing the user's motion, acting on his senses as well as computing the virtual experience in real-time. The immersion in virtual environment is evaluated through the user's perception and reaction. Virtual reality is used in a large variety of application domains which need multisensory interaction and navigation facilities. Virtual prototyping is also used in the industry to improve design process.
\end{abstract}

\section{INTRODUCTION}

Virtual reality is widely used in different application domains benefiting from the development of technologies coming from video game (as the computer does). Virtual reality consists in providing the possibility to interact with a virtual environment. The human is in the center of the application which needs dedicated technologies for interaction. Thus, knowledge of technologies and human factors are required to build an efficient virtual reality application.

This entry describes virtual reality principle and some key features existing in several applications. The first part shows how interactions occur in virtual environment. Principle of virtual reality involves knowledge of technologies and human factors because multisensory feedback interfaces need to be fitted with human senses as virtual immersion is evaluated on the human. The second part presents virtual reality technologies. Main sensory interfaces, which are constituted by hardware systems, are presented regarding the human sense they address. 3D representation of the virtual environment is then discussed regarding the virtual data management and the graphics process. User interaction techniques are performed in close link with sensory interface and are designed as function of the requirement of the application. The third part deals with perception of virtual immersion. The concept of presence is introduced to evaluate virtual immersion from the user point of view. Model and measurement of presence are discussed. Virtual reality is applied in a wide range of applications which require specifications in terms of interaction in virtual environment. The fourth part presents two important characteristics of interaction in virtual environment-multisensory interaction and natural user interaction. The fifth part deals with the navigation process in virtual environment. Two main cases of use are exposed - exploring the virtual world by miming walking, flying, or teleporting ways, and driving. A specific application for industry purpose is then discussed in the sixth part. Virtual prototyping aims at using digital representation in the design process of the object. The principle and main issues of virtual prototyping are exposed.

This entry is written to give an overview of virtual reality. Some general aspects are presented. The reader who wants to explore this topic in depth can find lots of rich information in several books.

\section{INTERACTION WITH THE VIRTUAL WORLD}

\section{Principle of Virtual Reality}

Virtual reality consists of software and hardware technologies which interact with the user to immerse him in a virtual environment. The principle is to create a relationship between the user and a virtual environment. For that, software technologies (computer graphics, real-time computing) as well as hardware technologies (human - computer interfaces) are required. Figure 1 provides an overview of the relationship between the user and a virtual environment. As described in Fig. 1, movements of the user are captured in real time in order to animate his avatar (virtual user), who interacts with the virtual environment. The effects of interaction between the avatar and the virtual environment are restituted to the user senses through sensory feedback technologies. This interaction loop has to be performed in the delay of the refresh rate of the excited human sense (commonly named as real-time process).

Then, virtual objects are perceived through technologies acting on human senses. These technologies are constituted of visualization devices, sound feedback, tactile and force feedback, as well as motion platform, for example. Interaction in virtual environment is realized through a relation between virtual environment and a virtual representation of the subject. A real-time interaction between the user and the virtual environment requires to capture motion or intention of the user in real time in order to 


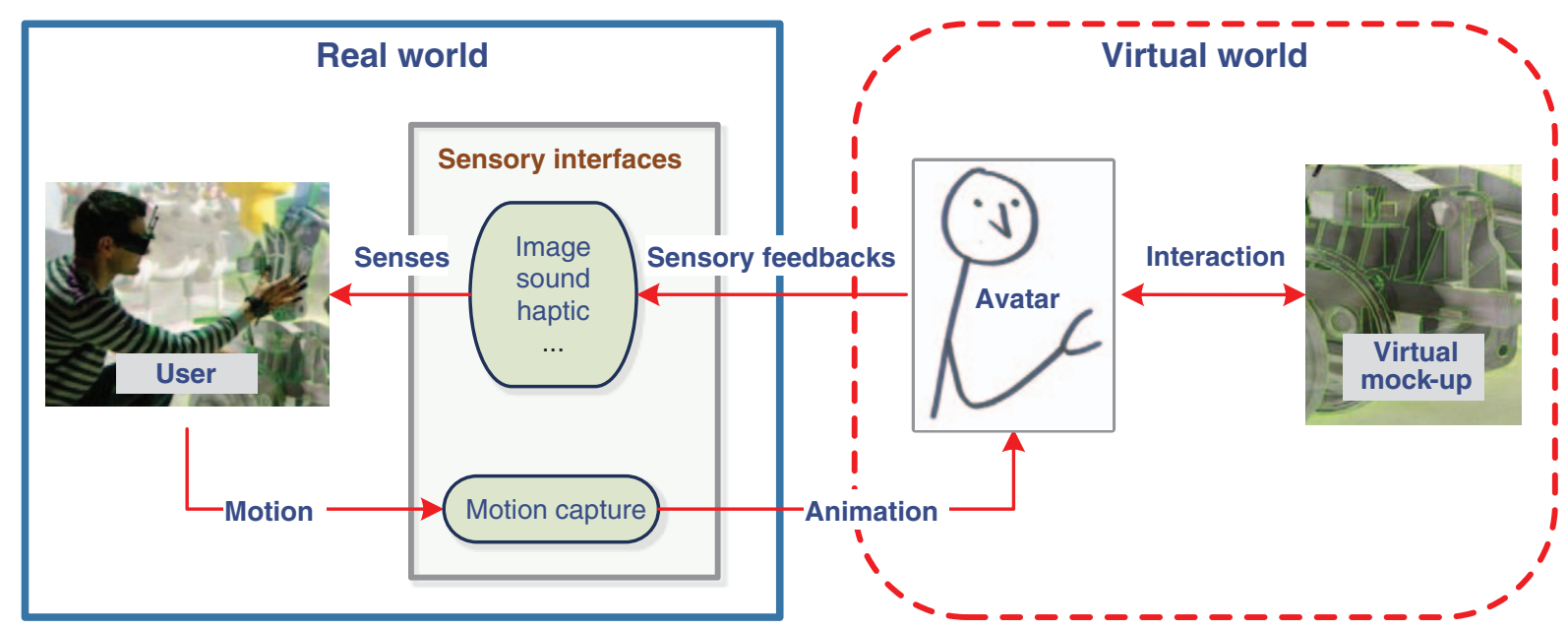

Fig. 1 Relationship between the user and a virtual environment.

refresh the avatar position and motion in the virtual environment according to the user's position and motion. In that way, a coherence between physical world and virtual world is realized in real-time.

The user's perception in virtual environment is evaluated through the concept of sense of presence which depends on the way the user can explore the virtual environment, the possibilities he has to interact with it, and the multisensory feedbacks provided by the technologies. Sense of presence is strongly dependent on the engagement of the body in the virtual immersion process. Thus, virtual reality tends to fully immerse the user in the virtual world as illustrated in Fig. 2.

\section{Multisensory Feedback Technologies}

Virtual immersion is enhanced by multisensory feedback acting on the user's senses. The knowledge of human senses characteristics enables the design and implementation of virtual reality technologies. Relationship between human

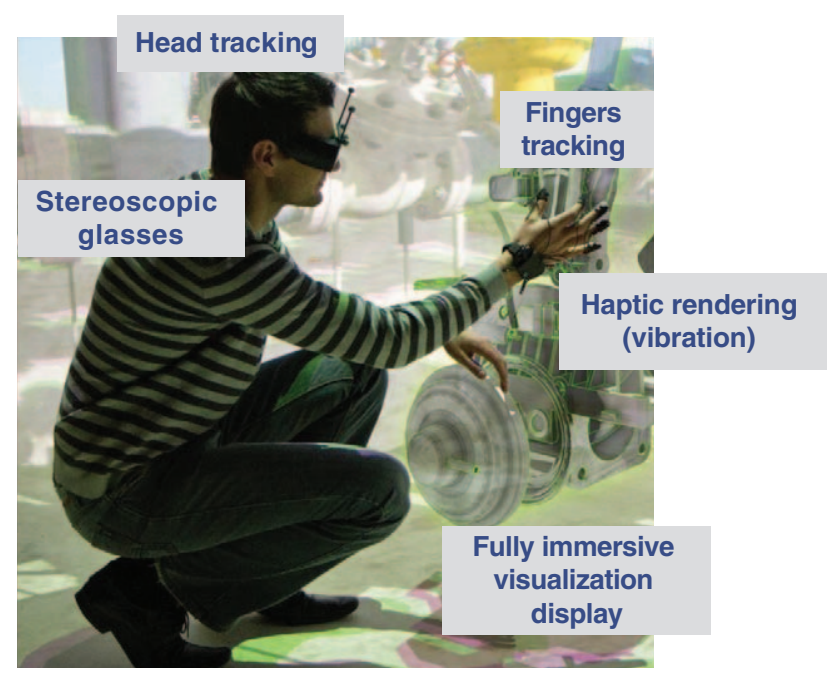

Fig. 2 Full immersion of the user in the virtual environment. senses and virtual reality technologies is optimized when the main characteristics of human senses are covered by technologies acting on it. Table 1 summarizes the main characteristics of human senses and its corresponding technologies which have to be considered for virtual reality applications.

Vision is one of the most important human sense to perceive the environment. Human eye is constituted of a lens (cornea and crystalline lens), a diaphragm (iris), and a light sensor (retina). Crystalline lens is warped by a rotational muscle for adapting the vision to the distance of the observed object. This warping enables the accommodation of the eye in relation to the distance of the object in order to produce a distinct image of the observed object. Due to the optical system (lens), the image produced at the retina level is not distinct for the whole scene but just around the area of the observed object. This area is called the depth of field from where all objects are distinct in the image. Images of the objects situated outside this area are blurred. The retina is composed of three different layers. At the light sensitive layer, retina has billions of photoreceptors which transform the luminous energy into electrical signal. A second retina layer (the inner retina) transforms electrical signal into different combinations for a first image processing operation (neighborhood effects by bipolar, amacrine, and horizontal cells). A third retina layer compresses the image information (on a wave train by ganglion cells) for a transmission to the brain. Two types of photoreceptors exist in the retina-rod and cone. Rods are highly sensitive to the light and give the capability to perceive at very low light conditions (moon light). Cones are sensitive to sun light and are mainly disposed in the central visual axis (foveal axis). Three different types of cones exist depending on the pigment used to transform light information into electrical signal. Each of the three pigments are sensitive to different wavelengths, enabling the perception of color. Luminous efficiency of rod is approximately one hundred times higher than the luminous efficiency of cone. Because rods and cones are not sensitive to the same level of luminance, vision 
Table 1 Relationships between human senses, main characteristics, and virtual reality technologies.

\begin{tabular}{|c|c|c|}
\hline Human sense & Main characteristics & Virtual reality technologies \\
\hline \multirow[t]{8}{*}{ Vision } & Sensitive between $400 \mathrm{~nm}$ and $800 \mathrm{~nm}$ & Dynamic screen \\
\hline & Huge range of brightness (from $0.0001 \mathrm{~cd} / \mathrm{m}^{2}$ to $10,000,000 \mathrm{~cd} / \mathrm{m}^{2}$ ) & Resolution capabilities \\
\hline & High resolution & Large FOV screen \\
\hline & High-contrast sensitivity & Eye tracking \\
\hline & $60 \mathrm{~Hz}$ per eye for VR (head motion) & Head-mounted display \\
\hline & Rotation speed up to $800^{\circ} / \mathrm{s}$ & \\
\hline & $180^{\circ} \mathrm{FOV}$ in horizontal plane & \\
\hline & $140^{\circ} \mathrm{FOV}$ in vertical plane & \\
\hline \multirow[t]{2}{*}{ Stereovision } & Stereoscopic rendering area (Panum zone) & Right/left channels splitting system \\
\hline & Visual accommodation and convergence relationship & Head-tracking system \\
\hline \multirow[t]{4}{*}{ Sound } & High dynamic & Ears tracking \\
\hline & Frequency range from $20 \mathrm{~Hz}$ to $20,000 \mathrm{~Hz}$ & Transfer function of user external ear \\
\hline & Binaural rendering & Real-time processing \\
\hline & & High-frequency rendering \\
\hline \multirow[t]{4}{*}{ Internal ear } & Acceleration sensor in space (rotation and translation) & Dynamic platform \\
\hline & Perception threshold in translation: $5 \mathrm{~cm} / \mathrm{s}^{2}$ & \\
\hline & Perception threshold in rotation: $2 \% \mathrm{~s}^{2}$ & \\
\hline & High sensitivity & \\
\hline \multirow[t]{2}{*}{ Tactile } & Skin sensors & Tactile feedback (vibration, electricity, etc.) \\
\hline & $1000 \mathrm{~Hz}$ frequency & \\
\hline \multirow[t]{3}{*}{ Kinesthesis } & Muscle sensors & Force feedback system \\
\hline & $1,000 \mathrm{~Hz}$ frequency & Treadmill \\
\hline & & Walking system \\
\hline
\end{tabular}

capabilities are not equivalent depending on the luminance. Different physiological research and evaluation on population lead to typical characteristics of the average human eye. Thus, the luminous efficiency of average human eye varies in the spectrum from $400 \mathrm{~nm}$ to $700 \mathrm{~nm}$ with a highest value at $500 \mathrm{~nm}$ in low-light conditions (due to rod) and at $550 \mathrm{~nm}$ in high-light condition (due to cone). Average human eye is then sensitive to a very large range of luminance (from $0.0001 \mathrm{~cd} / \mathrm{m}^{2}$ to $10,000,000 \mathrm{~cd} / \mathrm{m}^{2}$ ) from star light to sun light. The field of view is approximately $180^{\circ}$ on the horizontal plane and $140^{\circ}$ on the vertical plane. The perception of details in an image can be analyzed as the minimal angle under which the eye can distinguish two points. This angle defines the capability of eye resolution or visual acuity. Visual acuity depends on several factors such as the luminance, eccentricity, contrast, and motion of the observed object. Under the best luminous condition, the visual acuity on the foveal axis is close to an angle of $1 \mathrm{arcmin}$. This angle corresponds to 300 dots per inch for an image visualized at $30 \mathrm{~cm}$ or 45 dots per inch for an image visualized at $2 \mathrm{~m}$.

Stereoscopic vision given by the two eyes is activated by several coordinated muscles. This coordination makes the eyes converge on the object of interest. Because accommodation is performed on the object as well, a correlation between convergence of the two eyes and accommodation of each eye on object occurs. Thus, foreground and background of the observed object are blurred on image, which facilitate the fusion between the two left and right images and the analysis of the content by the brain. The object is then perceived as unique in the brain. Fusion of the two images of a same object in the brain is more difficult for a close object. A retinal disparity exists between the two images which is accepted by the brain for small differences. The fusion is still possible for images of objects situated in the area of the main object of attention (called Panum area). Accommodation and convergence contribute to the perception of depth in a scene. Accommodation acts on close objects (less than $1 \mathrm{~m}$ from the point of observation) and convergence acts on further objects (1-10 m under life conditions). At a distance beyond $10 \mathrm{~m}$ approximately, images from right and left eyes are quite the same so stereoscopy is not useful. Activations of each of these two phenomena are correlated. Accommodation induces a convergence mechanism for a close object, for example.

The visualization of a virtual environment in stereoscopy requires several steps. A first step is to create two synthetic images from a point of view on the virtual environment. Two virtual cameras are used from a right and a left virtual eye positions. These two images are rendered and visualized on a stereoscopic screen with a dedicated system to split images 
between right and left eyes. Many different systems exist and most of them use flat screen without any eye convergence tracking system. So, they do not guarantee the correlation between accommodation and convergence. That is the most important difference between stereoscopy in real world and stereoscopy in virtual world. Then, an important disparity between images may occur which can cause eye discomfort. Previous studies ${ }^{[1]}$ showed that the visual system can fusion object images for horizontal parallax less than $1.5^{\circ}$ and for vertical parallax less than $0.4^{\circ}$. Some rules have to be followed to avoid these side effects. For example, the principal object for the application has to be situated at the level of the screen. The two virtual cameras used to render the stereoscopic image for the user have to be situated in parallel axis at inter-ocular distance between each other for a scale 1 rendering.

All the excited senses collaborate during the virtual immersion experience. This collaboration is reflected by a sensory fusion made by the brain to produce information. Depending on the task, the sensory fusion can lead to an average signal resulting on several senses or a preference for one sense. A conflict between several modalities can cause headache or cyber sickness in virtual environment. The human is part of the whole system and may adapt his movement depending on the system (important latency, for example).

\section{Virtual Reality Issues}

The user is engaged in the immersion process so that his perception results of an alchemy between his engagement, the technologies, and the task. Thus, the whole system composed of the human and the technology has to be taken into account to design a virtual reality application. Consequently, key factors to consider in the virtual immersion are human factors, technologies, and the application as described in Fig. 3. These key factors involve scientific and technological issues jointly addressed by a scientific community focused on technology (computer graphics and mechatronics) as well

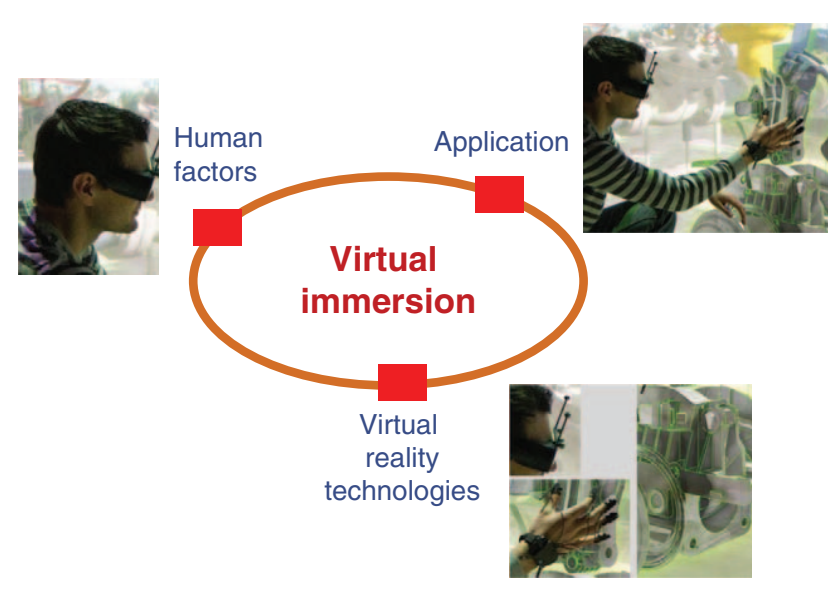

Fig. 3 Key factors for virtual immersion. as a scientific community on human factors (ergonomics, neuroscience, cognitive science). Sense of presence may be influenced by these following main parameters: (a) interactivity of the system in the function of the user's motion, (b) multisensory feedbacks and quality of the sensory coupling, (c) engagement of the subject in the virtual environment, and (d) ecological interfaces.

\section{VIRTUAL REALITY TECHNOLOGIES}

Interaction between a user and a virtual environment requires several different technologies both in the virtual world and in the real world. The majority of these technologies belong to computer science, human-computer interfaces, as well as mechatronics.

\section{Sensory Interfaces}

\section{Motion Tracking}

Motion tracking technologies are used to animate the user's avatar at the same time and in the coordination of the user's motion. These technologies aim at informing the computer on the motion of the user. The goal is to adjust the reference of the virtual world with one of the physical world. Several types of technologies exist based on mechanical system, electromagnetism, ultrasound, or image. The part of the body which is needed to be tracked in real time depends on the application. The most important part of the body to be tracked is eye position in the physical environment because vision is often the first sense used by virtual reality. Eye-tracking technologies can be used to guarantee the best possible adjustment of virtual cameras on each eye (for a good convergent rendering). But, because of their complexity and the high speed of eye motion (more than $800^{\circ} / \mathrm{s}$ ), eye-tracking systems are not highly utilized. Most of the time, virtual reality systems use head tracking instead of eye tracking. Right and left eye positions are estimated from head position and enable to place one virtual camera for each eye to render the virtual image according to the user's position in the virtual environment. Another important part of the body to be tracked is the hand in order to interact with the virtual environment. The choice of the technology depends on the type of interaction technique (if the interaction technique is more abstract or more natural). For more natural interaction technique, hand or fingers tracking systems or data glove can be used. For more abstract interaction technique, several technologies from CAD industry or video games can be used (e.g., joystick, gyropoint, wand, etc.). If the full body needs to be tracked, dedicated technologies such as real-time full body-tracking suit, real-time motion-tracking components placed on body joints, and image-based motion tracking systems exist. These technologies come from the video game industry and entertainment or film industry. 


\section{Visualization Devices}

Visualization devices use different technologies to produce image. They can use image projection on screen (based on LCD and lamp projector or laser projection) or electronic screen (based on LCD or OLED technologies). The characteristics of screen technologies have to be adjusted according to the human eye capabilities. Because eye resolution is close to 1 arcmin, it requires to display image with a corresponding resolution. For example, a $4 \mathrm{~K}$ screen (UHD format) displayed on a $3 \mathrm{~m}$ large screen observed at a distance of $2 \mathrm{~m}$ is equivalent to a resolution of $1 \mathrm{arcmin}$ from the eye point of view. The human visual system has a remanence of approximately $30 \mathrm{~ms}$ which requires to produce image at a frame rate of $25 \mathrm{~Hz}$ for monoscopic view ( $50 \mathrm{~Hz}$ for stereoscopic view). But, if the user is in motion in front of the screen (to move around a virtual object, for example), fast head motion has to be considered. Then, video frame rate has to be up to $60 \mathrm{~Hz}$ for monoscopic view (120 Hz for stereoscopic view). Visual human system is sensitive to a very high range of luminance (from 0.0001 $\mathrm{cd} / \mathrm{m}^{2}$ to $10,000,000 \mathrm{~cd} / \mathrm{m}^{2}$ ). Unfortunately, actual screens cannot render image with this requirement (best screens can render luminance only from $0.001 \mathrm{~cd} / \mathrm{m}^{2}$ to $500 \mathrm{~cd} / \mathrm{m}^{2}$ ). Dedicated techniques can be used to give the user the illusion of a high range of luminance on image (with the use of high dynamic range image management system to generate image and tone-mapping techniques to render image).

The first family of visualization system consists of static screen in front of which the user stands. Screens can be of different shapes and sizes. Large screens are useful to render virtual environment at scale one for user so they can visualize, navigate, and interact in the virtual environment as they would do in the real one. They can have the shape of a large wall or a big cube (as the CAVE system) or rounded wall (as the reality center) or a sphere (as screen dome). Virtual desk consists of a horizontal screen at a desk configuration enabling the user to stand in front of middle-size virtual objects (for design purpose, for example). Virtual desk can have an added vertical screen behind the desk to give a better immersion to the user. Small-size screens can be used as well for specific purposes. Thus, the use of PC tablet or smartphone can propose a virtual window view through the world to give added information to the user in a real or virtual world. In using the camera of the device, an augmented reality view can be proposed to combine the view of real world with elements of virtual environment. To render stereoscopic vision using static screen, a dedicated system to attribute left image to left eye and same for right side is needed. Some of these technologies filter the light by spectral band or light polarization. The process invented by d'Almeida in 1858 separates left and right image flows by the use of a mechanical occluding system. Based on this principle, new technologies use electronic shutter placed in front of each eye and functioning in switching with the image display. These technologies require high-frequency display. The process invented by Berthier in 1896 is composed of a physical grid, making the assignment of each channel to each eye. New technologies used this principle to use electronic mask between backlight and LCD screen. Another process invented by Bonnet in 1939 uses a lenticular network placed in front of the screen enabling the assignment of channels by the refraction of the light through this little lenses. New technologies use this principle in front of LCD screen. Berthier and Bonnet processes do not need any glasses to render stereoscopic view unlike polarization or occlusion principles.

The second family of visualization system is constituted of head-mounted display. Screens surround eyes and an optical system is used to produce distinct image for the eye. Such technology does not need the system to split right and left channels because each eye can directly see its own dedicated image. Two types of head-mounted display exist. The first type is closed and the user sees only the virtual environment. This virtual reality, head-mounted display fully immerses the user in the virtual environment as he cannot see the physical environment. So, they tend to isolate the user from the real world and can disturb his perception if the other senses are not excited in the same way. A second type of head-mounted display allows the user to see through the display and combine a virtual image to the view of the real scene. This type of device is also called augmented reality device for that reason.

A third family of visualization system corresponds to volumetric image. One technology consists of volumetric image created by a series of images projected through an opto-mechanical rotated system. The range of projected images is distributed in the space by a rotating screen and display a volumetric image. Another technology is constituted by holography. The process was invented by Denis Gabor in 1947 and consists in capturing the phase as well as the amplitude of the luminous field and restituting it to the user by a laser light. This technique is well known for static image and several technologies are in progress for dynamic images.

\section{Haptic Feedback}

When interacting with a virtual environment, the sense of contact with a virtual object may be interesting to render as function of the application. For that, force feedback technologies may be used. Technologies come from the robotics domain and can take different shapes and complexity. Robot arm uses several joints enabling the robot to follow the motion of the user's hand and control this motion according to collision between the hand's avatar and the virtual object. Robot can be external to the user (the user takes the robot's arm to control his hand's avatar) or can be more integrated to the user (as exoskeleton). To enable the user to perceive texture, tactile feedback can be performed in using vibrations, pin matrix or pressure rendering systems. ${ }^{[2]}$ Some technologies can render heating as well. 


\section{D Sound}

The use of $3 \mathrm{D}$ sound in virtual reality applications improve the immersion of the user in bringing added value when interacting with virtual objects as well as audio ambiance in the scenario of the application. Because of the difficulty to compute a synthetized 3D sound in real time, 3D audio has been introduced in virtual reality in using existing real sound and spatializing it in virtual environment. Thereafter, it has been used as a feedback in case of interaction with the virtual object. The $3 \mathrm{D}$ sound has to be precise in order to allow the user to identify the localization of the sound event in virtual space. Human hearing depends on several factors as the slight difference on perceived sound for both ears in terms of intensity (inter-aural level difference) and time delay (inter-aural time difference), the effect of human body and human listening system (which can be experimentally measured and modeled by the head-related transfer function), and the environmental acoustics (reverberation and reflections of the sound on different parts of the environment). Technologies to render 3D sound in virtual environment are constituted by real-time head-tracking system, a headphone system or multichannel systems, and real-time computing 3D sound system (software and hardware). Softwares dedicated to real-time computing of 3D sound are based on image-rendering principles. To improve the precision of sound localization, sound metaphors can be designed as an added value for the sound perception.

\section{Motion}

Motion is perceived through several senses such as vision, kinesthesis, and vestibular system. Kinesthesis feedbacks consist of on sensors situated on the basis of muscles which feed information to the brain on the articulation states. To excite kinesthesis, virtual reality technologies provoke the physical motion of the user. Many different systems exist in that goal to make the user walk (treadmill in front of a screen, pressure sensors on shoes to physically walk when using a head-mounted display, virtusphere device, circularfloor device) or bicycle (bicycle in place). Vestibular system gives information on acceleration (in rotation and translation) and can be stimulated with the use of motion platform system (commonly used for driving simulation, for example).

\section{D Representation}

\section{Data Management}

Digital mock-up is a set of data representing the object to visualize. The virtual object is described by its shape, material, and several physical properties (e.g., mass, articulations, etc.). Shape description uses techniques of elementary and differential geometry. Common techniques represent a virtual object by boundary representation or constructive solid geometry. The use of polygonal plane facets is both simple and costly because it induces a detailed sampling surface for round objects. Cubic patches enable a fine representation with a relatively low number of patches to represent an object. B-Spline representation (and particularly Beziers surfaces) are commonly used because of its interesting features (as continuity capability, invariance to linear transform, defined by control points, etc.). Nonuniform rational basis splines (NURBS curves) are equivalent to generalized B-Splines and enable easy manipulation for free-form generation as well as easy computing of intersections between objects. This description, commonly used in computer-aided design (CAD) domain, is often converted in polynomial description and specifically in triangles to facilitate the real-time management of the data for graphics rendering. In industry, digital mock-up is the central element of the design process. It is comprehensive and can describe geometry (macro and micro), material, as well as assembly operation. Digital mock-up is involved in the whole life cycle of the product and used by different actors along the design process (architecture, thermal analysis, mechanics, manufacturing, etc.).

Virtual mock-up is dedicated to real-time interaction in virtual reality. It is adapted to the sensory feedback and the task. For example, from a unique digital mock-up, two virtual mock-up might be generated-one virtual mockup dedicated to visualization and another one dedicated to haptic feedback. Virtual mock-up is managed by computer. Considering the actual computer architectures, geometrical data are usually realized on the basis of triangle representation. Computations are simplified but numerous. The generation of the virtual mock-up from its native digital mock-up is guided by such criteria as the application task and user interaction. Several methods and tools can be used in that goal as simplification of geometry, adapted simplification dedicated to sensory feedback, adaptation of the virtual mock-up to the interaction process, or interaction with the lighting. Thus, the transition from digital mock-up to virtual mock-up consists generally of transforming the mathematical surface in a set of triangles to represent the virtual object. Several existing methods are based on the Delaunay triangulation or the Voronoi diagram. This transformation induces loss of information on the topology. Depending on the triangulation method, holes may appear in the surface which necessitate a specific operation to keep the coherence of the surface. In function of the chosen threshold, the triangulation operation can create a representation with too many triangles to be managed in real time. The virtual mock-up has to be simplified then. Several methods exist in the literature. A first family consists of suppressing some entities of triangles in an iterative way (delete vertices, fusion of edges or facets). A second family consists of modifying the triangles guided by a model (surface subdivision, curvature criteria).

\section{Computer Graphics}

Visualization of virtual mock-up is performed through graphics rendering consisting of creating a synthetized 
image from a user's point of view. The created image is the result of a simulation of the interaction between virtual lighting and virtual mock-up (characterized by its material and shape). Light-material interactions can be very complex to represent depending on its features (reflection, refraction, caustic effect, multiple reflections, etc.). Different models have been developed to express these effects. ${ }^{[3]}$ The surface can be modeled by a succession of micro facets with orientations and sizes function of its features (as roughness). Material can also be modeled by the way its surface modifies the trajectory of the light with the use of a bidirectional reflectance distribution function. This function can be expressed by simplified mathematical model or can be considered as a scalar for matte surface. To produce an image, the camera is usually a pin-hole model. This model enables the simulation of trajectory of the light rays from the camera instead of lighting to optimize the rendering time. Depth of field effect cannot be rendered with the use of the pin-hole model and so added signal processing may be computed on the image in that goal. Ray tracing technique is used to render the path of the light through different materials of the virtual scene and can then express reflection and refraction effects. The simulation principle is quite simple but can take time because of the numerous computations to be performed. Depending on the complexity of the scene, the materials, and the computer's power, the real-time rendering can be challenging with the use of ray-tracing technique. Another complementary method which can render matte surface lighting effect is the radiosity technique. This technique is a global illumination technique inspired by the domain of thermal exchange. The scene is decomposed into small matte surface elements which exchange lighting energy with each other. Because the scene is represented by matte surface elements, the luminance at each point of the scene is independent from the point of view, making this technique very useful for real-time visualization after an off-line rendering step. A complementary technique to improve the realism of the image is to use texture which consists of applying a pattern on the surface of the virtual object. Texture can restitute details, lighting, as well as roughness of a surface and so the details on the image are on the pattern instead of the 3D model.

\section{User Interaction Techniques}

\section{Navigation}

Navigation in virtual environment requires devices both for controlling the motion of the user's avatar and for perceiving the motion. Regarding the application requirement, different types of technologies can be used. As mentioned previously, walking or biking systems can be used. Devices coming from the video game domain (as gamepad) or motiontracking technologies can also be utilized. These navigation devices are used with dedicated navigation technique to control the navigation parameters (e.g., speed, acceleration, translation, rotation). Thus, different techniques (more or less intuitive) can be chosen for the same device. Walkingin-place technique proposes the user to mimic the motion of walking when standing in place on a force measurement platform. Grab-the-air is a technique where the user has to pull the virtual environment to him by gestures in the air. Gogo navigation technique is controlling a virtual hand in the virtual environment to guide the direction of navigation. Route-planning is a technique where the user points his path in the virtual environment and his motion is computed according to the path. Target navigation proposes the user to point the destination and teleporting him to the designated location. Virtual companion technique gives the user the control of a virtual companion to move around in a virtual environment. The user's avatar is attached to the virtual companion during navigation (by virtual rope).

Several classifications can be found in the literature. ${ }^{[4]}$ Table 2 proposes a classification of different navigation techniques according to two different considerations. The first consideration belongs to the way the action is realized. If the action is realized directly from the avatar, the interaction process is made in an egocentric way (coming from the user's reference). If the action is realized from the virtual environment, the interaction process is made in an exocentric way (external from the user). The second consideration belongs to the nature of the interaction process. In the case of a natural interaction process, the interaction is considered as concrete. In the case of the interaction process requiring a specific learning process, this interaction is considered as abstract.

\section{Manipulation}

Manipulating the virtual object is another important task the user needs to perform in the virtual environment. The user manipulates the virtual environment to select and change the position of the virtual object or to act on different features of the virtual object. As for navigation techniques, several manipulation techniques can be used depending on the task and the device. Virtual hand is a virtual extension of the user's arm to interact (beyond the peripersonal space) in the virtual environment. Virtual pointer enables the user to reach the virtual object in the virtual space as he would do in 2D using mouse control. Ray casting consists of a virtual ray controlled by the user

Table 2 Navigation techniques.

\begin{tabular}{|c|c|c|}
\hline $\begin{array}{l}\text { Navigation } \\
\text { characteristics }\end{array}$ & $\begin{array}{c}\text { Concrete } \\
\text { navigation } \\
\text { technique }\end{array}$ & $\begin{array}{c}\text { Abstract } \\
\text { navigation } \\
\text { technique }\end{array}$ \\
\hline \multirow[t]{3}{*}{ Egocentric technique } & Walking in place & Gamepad \\
\hline & Treadmill & Virtual car \\
\hline & Stationary bicycle & Grab the air \\
\hline \multirow[t]{3}{*}{ Exocentric technique } & Go-go navigation & Virtual companion \\
\hline & & Target navigation \\
\hline & & Route planning \\
\hline
\end{tabular}


and interacting with the virtual objects over the distance. Aperture technique enables the user to adjust the precision of interaction with the virtual object. Image plane technique is a representation of the virtual environment using a $2 \mathrm{D}$ projection. Voodoo doll technique uses a miniature copy of the object to facilitate the interaction process. World-inminiature replicates the virtual environment in miniature to interact with it. Virtual menu as virtual tablet is $3 \mathrm{D}$ adaptation of the $2 \mathrm{D}$ menu to interact with the object. ${ }^{[5,6]}$ Table 3 proposes a classification of different manipulation techniques with the same typology as the one used for navigation techniques. Of course, different techniques can be combined to accomplish a task. For example, the user can select the virtual object with one technique and manipulate it with another one. Using such hybrid method enables the user a larger degree of freedom on object manipulation but necessitates to take care of compatibility between the techniques used during the same task as well as learning requirement.

\section{Tactile Interfaces}

The large diffusion of tactile interfaces through smartphone and tablet promoted the practice of finger interaction language. In touching a sensible interface with fingers, a user can interact with a virtual environment for navigating or manipulating an object. Several types of gestures are common for zooming, rotating, moving forward or backward, catching, and so on. These interaction techniques can also be transposed in a fully immersive virtual reality technology.

\section{PERCEPTION OF VIRTUAL IMMERSION}

\section{Model of Perception}

Concept of presence has been proposed as an indicator of the virtual immersion since the beginning of virtual reality technology. The "Sensorama" virtual theater developed by Morton Heilig in the 1950s introduced the notion of "being there" in a mediated environment. Several models of perception have been proposed in the literature in order to define and measure the perception of virtual immersion.

Table 3 Manipulation techniques.

\begin{tabular}{lll}
\hline $\begin{array}{l}\text { Navigation } \\
\text { characteristics }\end{array}$ & \multicolumn{1}{c}{$\begin{array}{c}\text { Concrete } \\
\text { manipulation } \\
\text { technique }\end{array}$} & $\begin{array}{c}\text { Abstract } \\
\text { manipulation } \\
\text { technique }\end{array}$ \\
\hline $\begin{array}{l}\text { Egocentric } \\
\text { technique }\end{array}$ & Virtual hand & Virtual pointer \\
& Ray casting \\
Exocentric & Image plane technique & Aperture technique \\
technique & Voodoo doll in miniature \\
& & Virtual menu \\
& & Virtual tablet \\
& & Naming \\
\hline
\end{tabular}

The important of these models come from the psychology domain. Bruno Herbelin ${ }^{[7]}$ used the neo-behaviorism psychological theory (where the behavior depends on the chain stimulus-organism-response) to consider the presence as the result of a mediation chain as well as a psychological mechanism. Franck Biocca ${ }^{[8]}$ proposed to consider the body as a communication device included in a technological chain, mediated by computer. Depending on the extent to which the body is linked with technology, the human may accept these technologies as a part of himself (as a cyborg accepting prosthesis). The acceptance process depends on the naturalness of technologies and mind work during the learning process. Matthew Lombard ${ }^{[9]}$ defined presence as what happens when the participant forgets that his perceptions are mediated by technologies.

\section{EVALUATION OF VIRTUAL IMMERSION}

Virtual immersion can be evaluated according to the chosen model of perception. Reactions of the body during or after the virtual immersion exposure give a first indication on the sense of presence. Physiological feedback or task performance can be objectively measured in that objective. Various measurements can be performed-heart rate, myography activity, encephalography activity, breathing, sweating, eye motion, speaking, or postural sway. Cyber sickness can be evaluated through a combination of objective measurements such as heart rate, eye movement, and postural sway, for example. As well, consequences of the user experience can be subjectively evaluated through questionnaires as the one proposed by Maria Sanchez-Vives ${ }^{[10]}$ on the sense of presence. In some cases, cyber sickness (caused by sensory conflict) may occur. Robert Kennedy ${ }^{[11]}$ developed a simulator sickness questionnaire in that context. Determinants of presence are numerous. Jonathan Steuer ${ }^{[12]}$ proposed to consider the extent and fidelity of sensory information, the real-time synchronism, content factors (as event, virtual representation of the body) and user characteristics (perceptual, cognitive, and motor's abilities).

\section{INTERACTION IN VIRTUAL ENVIRONMENT}

\section{Multisensory Interaction}

In combining several sensory modalities, the virtual immersion can be improved. The design of a virtual reality system requires a sufficient knowledge on human senses characteristics for adapting technologies to application. Depending on the task, a sense can be preferred from another. ${ }^{[13]}$ For example, vision is the main sense used to join two objects in space for ballistic task and haptic feedback is used for an assembly task between two parts of an object. Thus, the interaction design process has to take into account the different tasks of the virtual experience. Multisensory interaction is widely used in the industry domain (e.g., for design 
process, maintenance, training, assembly assessment), health domain (e.g., surgery training, therapy, gesture training), military domain (e.g., training), or scientific domain (e.g., big data interaction).

\section{NATURAL USER INTERACTION}

Several interaction technologies and techniques tend to propose intuitive approach for the user and are commonly named as natural user interaction. The first type of natural user interaction proposes the user to interact in the same way he does in the real world. The gestures required by the user to accomplish a task would be the same in the virtual environment as in the real world. Interaction techniques and technologies have to be adapted to these requirements for this goal. A second type of natural user interaction considers user experience and common practice with human-computer interaction, as mouse device, joystick interface, tactile interaction process in using smartphones (with specific interaction language based on finger gestures). Natural user interaction is used in the video game domain, industry, entertainment, and health domains where users are non-specialists.

\section{NAVIGATION IN VIRTUAL ENVIRONMENT}

\section{Exploring the Virtual World}

Exploration of the virtual environment is an important task for a large number of domains using virtual reality. Some scientific and technical challenges remain to restitute motion perception to a user in virtual reality. In the case of using a static display (the case of large screen' for example), the user cannot make huge displacements. While the user can move in real world, he has to stand in front of the screen in virtual reality. So dedicated systems have to be proposed in order to produce a motion perception. Usually, a compromise has to be done in the experimental set up design between complexity of the technology and motion perception rendering. Walking is one of the most natural human motion. The movement of each human joint during the motion is very complex as it is correlated to the other joint movements for a specific task (learning effect) under the constraint of gravity. Many parameters occur during walking which impact balance and postural control. Walking motion is possible in using treadmill technologies. ${ }^{[14]}$ Simple unidirectional treadmill enables the user to walk along the direction of the treadmill. In combining several unidirectional treadmills, a 2D treadmill can be built (some prototypes exist in research laboratories). As treadmills, other systems enable the user to walk while staying at the same position relative to the reference of the screen. In the Virtusphere system, the user is inside a sphere rolling on site. The user can see the virtual environment through a head-mounted display. Another system, proposed by Hiroo Iwata, ${ }^{[15]}$ is constituted of several robots on which the user walk and which make him stay static in the room. Some drawbacks are present in the use of these systems. Because of the inertia of the system, stopping suddenly or turning may induce posture imbalance. These systems have to be used with care (limited speed, low accelerations, safety harness). Another technique uses the principle of walking in place. A force sensor placed under the feet (on shoes) or using a force platform brings information on alternative pressure under feet. The user is in the front of the screen and realizes movement of trampling, giving him the illusion of walking.

In using head-mounted display, the user can freely move in the physical space. The user wears a head-mounted display and a motion-tracking system can capture the position of the user in the space. But, in the real environment where the user moves are huge, it is finite because of limitations of the motion-tracking capabilities. To give the illusion of moving in an infinite space, technique of redirected walk has been proposed. ${ }^{[16]}$ In this technique, small visual motions in translation and rotation are done continuously in order to replace the user in a limited physical area. These small motions are done with speed and acceleration which stay under human perception threshold. ${ }^{[17]}$

During exploration task in the virtual environment and depending on technologies, motion sickness may occur. Exploration task is widely used in several application domains such as virtual building, cultural heritage, big data exploration, etc.

\section{Driving}

In driving, the user is in interaction with his vehicle. His own motion is perceived both through visual information by the vection in the peripheral field of vision and through the vehicle's motion stimulating internal ear. Thus, the sensory information comes from the visual channel combined with vestibular channel. Previous studies showed that a stimulation of the vestibular channel improves the perception of simulated driving in limiting the motion sickness. This vestibular channel stimulation is realized by the movement of the user with a dynamic platform. Driving simulation requires a fully virtual immersion because the motion of the body has to be rendered finely. While driving, the driver is in contact with the steering wheel, pedal system, and the seat. Thus, sensory feedback as vision, haptic pedal on the feet and steering wheel as well such as vestibular system (acceleration rendering) have to be performed regarding the virtual car behavior controlled by the driver. If the cockpit is consisted of a physical device representing a car, the virtual environment can only be the road and its environment. In this case, the driver has a far-reaching view (more than $10 \mathrm{~m}$ ) and a stereoscopic rendering is not required. If the cockpit is partially physical (consisting a steering wheel, pedals, and seat) and virtual (other parts of the car), the driver experiments his driving by a far-reaching view (road) and a close view (virtual cockpit), which requires stereoscopic rendering. As the field of view has to 
be wide in order to restitute the vection effect to the driver, the display has to surround the driver. Software architecture in driving simulator is constituted by several modules for virtual car simulation, sound rendering, haptic feedback on steering wheel and pedals, and visualization feedback as motion platform.

Many driving simulators use dynamic platform based on the mechanical architecture using parallel arms. Parallel platforms have capabilities to manage important loads while having good precision in position. The parallel platform proposed by Gough and Stewart in 1970 is a platform operated by six actuators having six degrees of freedom. The control of the platform motion is realized in modifying the length of the actuators. A model of the platform enables to infer inverse cinematic for the control of the platform. Such an inertial platform cannot provide large longitudinal acceleration due to its architecture. The tilt coordination technique is able to restitute such longitudinal acceleration when using parallel platform. This technique consists of tilting the platform compared to the vertical axis. The user perceives the vertical axis in his own spatial reference, which is the inertial platform. Thus, in the spatial reference of the platform, gravity can be decomposed into two components-a component perceived as the vertical by the user and a component perceived as a longitudinal acceleration by the user. To prevent the user to perceive the tilt coordination motion, the inertial platform has to be moved to its tilt coordination position with a tilting speed below a perception threshold of the vestibular system. This perception threshold is estimated at an approximate value of $3 \% \mathrm{~s}$. To facilitate this sensory illusion, the visual perceived information has to confirm to the user his position regarding the spatial reference of inertial platform. For this, visualization has to be computed in accordance with the movements of the platform. This correlation can be performed by modifying in real time the image in function of the motion of the platform, if the screen is static. Another solution is to place the screen on the inertial platform to avoid real-time computing of the image according to the platform movement. The control of the platform has to be done in accordance with the perception criteria and the degrees of freedom of the platform. Four major motion platform strategies can be found in the literature towards this. (classic, adaptive, optimal, predictive). The Gough-Stewart platform can be associated to a rail system in order to extend the degrees of freedom of the whole system (to reproduce lateral acceleration in overtaking manoeuvres, for example). Other driving simulators based on different mechanical systems such as an arm-robot supporting the driver's cockpit or centrifuge system to better render accelerations for long time exist.

Driving simulator is used for a wide range of applications. For example, in the industry, driving simulation is used to assess advanced drive assistance system during the design process of a vehicle. It can also be used to study the design of semi-automotive vehicles and the cooperation between the user and the vehicle during critical situations.
In the video game domain, driving simulation is widely used mostly on the basis of static simulator and for some with dynamic platform.

Because the driving task stimulates both visual and vestibular channels, the sensory fusion can be difficult to operate with the use of basic technologies, and motion sickness may occur.

\section{VIRTUAL PROTOTYPING}

In the industry domain, especially in large companies, virtual reality is a tool that is becoming commonly used in several stages of the product life. Virtual prototyping consists of designing the product, reviewing the product with several people, as well as evaluating the product in virtual environment. Digital mock-up is the core material of virtual prototyping enabling engineers to design the product before manufacturing it. In the industry (especially small and medium enterprises), evaluation is performed with physical prototyping, as described in Fig. 4. A major issue in the industry is to evaluate the product based on its virtual representation. Virtual reality is then used to enable engineers to take decision in the virtual environment as they would do in real world based on physical prototype. Figure 5 illustrates the validation process in using virtual prototyping with

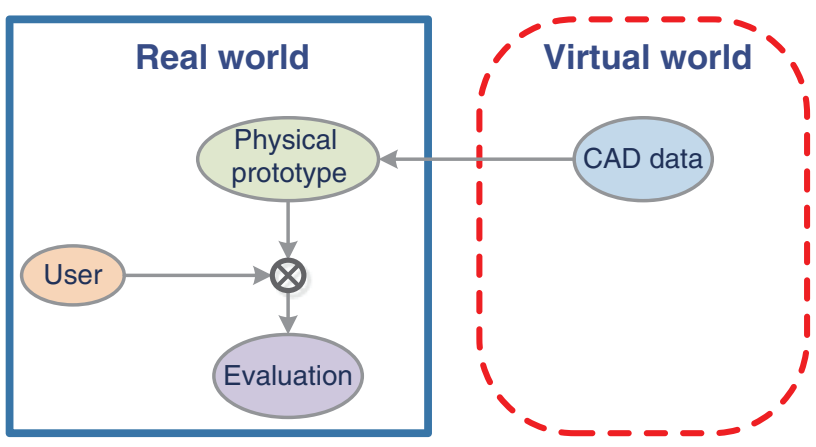

Fig. 4 Physical prototyping.

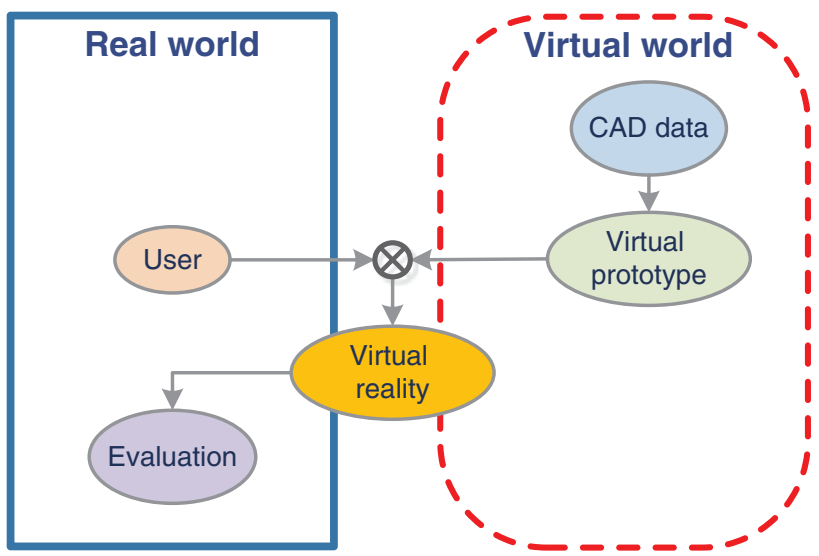

Fig. 5 Virtual prototyping. 


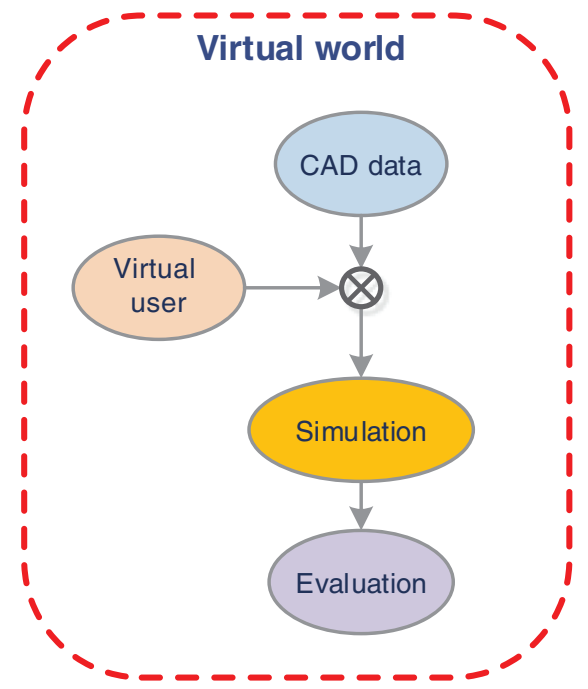

Fig. 6 Virtual evaluation.

virtual reality technologies compared to the one done with physical prototype. This process is actually performed in the industry, and some virtual reality applications provide a more efficient design in terms of time process and evaluation of a multitude of parameters compared to a costly experience plan by simulation. The added value of virtual reality for prototyping is due to the fact that virtual the reality process puts the experts in the loop and the whole process benefits from the expertise of humans. In the future, with more knowledge on the brain and decision-making process, a virtual expert might be able to propose a complete virtual evaluation process as illustrated in Fig. 6.

\section{CONCLUSION}

Virtual reality technologies offer a huge amount of possibilities to the user to interact with the virtual environment. Several competencies are necessary to build virtual reality applications (computer graphics, human computer interfaces, mechatronics, ergonomics, etc.). Virtual reality technology developed for a dedicated application is specific because each application has its own scenario. Thus, virtual reality application is the result of digital craftsmanship. A very few guidelines exist, and work-based experience is important to develop a virtual reality application as a strong knowledge is required to choose the best fitting between technologies and applications. Nevertheless, some criteria which can lead to a good interaction technique are linked to the user performance, user comfort, intuitiveness, and an easy-to-learn interaction technique as well as the effectiveness for the task. Lots of challenges remain in the future. Some of them are related to multisensory augmented reality, holography, interaction process with big data or collaboration over the distance between asymmetrical virtual reality technologies.

\section{REFERENCES}

1. Fuchs, P.; Moreau, G.; Guitton, P. Virtual Reality: Concepts and Technologies; CRC Press: Boca Raton, 2011, $432 \mathrm{pp}$.

2. Visell, Y.; Duraikkannan, K.A.; Hayward, V. A device and method for multimodal haptic rendering of volumetric stiffness. In Haptics: Neuroscience, Devices, Modeling, and Applications, Part-I; Auvray, M., Duriez, C., Eds.; Springer: Berlin Heidelberg, 2014; 478-486.

3. Foley, J.; van Dam, A.; Feiner, S.; Hughes, J. Computer Graphics: Principles and Practice; Addison-Wesley: Reading, 1990; 1174 pp.

4. Bowman, D.A.; Kruijff, E.; LaViola, J.J.; Poupyrev, I. 3D User Interfaces: Theory and Practice; Addison-Wesley Professional: Redwood City, 2004.

5. Dachselt, R.; Hübner, A. Three-dimensional menus: A survey and taxonomy. Comput. Graph. 2007, 31 (1), 53-65.

6. Lemoine, P.; Vexo, F.; Thalmann, D. Interaction techniques: 3D menus-based paradigm. In Workshop on Augmented Virtual Reality, Sept 2003; University of Geneva: Geneva, Switzerland.

7. Herbelin, B.; Vexo, F.; Thalmann, D. Sense of presence in virtual reality exposures therapy. In 1st International Workshop on Virtual Reality Rehabilitation, Lausanne, Switzerland, Nov 7-8; 2002.

8. Biocca, F. The cyborg's dilemma: Progressive embodiment in virtual environments. J. Comput-Mediat. Comm. 1997, 3 (2), 12-26.

9. Lombard, M.; Ditton, T. At the heart of it all: The concept of presence. J. Comput-Mediat. Comm. 1997, 3 (2) online.

10. Sanchez-Vives, M.; Slater, M. From presence to consciousness through virtual reality. Nat. Rev. Neurosci. 2005, 6 (4), 332-339.

11. Kennedy, R.; Lane, N.; Berbaum, K.; Lilienthal, M. Simulator sickness questionnaire: An enhanced method for quantifying simulator sickness. Int. J. Aviat. Psychol. 1993, 3 (3), 203-220.

12. Steuer, J.S. Defining virtual reality: Dimensions determining telepresence. J. Commun. 1992, 4, 73-93.

13. Ernst, M.O.; Banks, M.S. Humans integrate visual and haptic information in a statistically optimal fashion. Nature 2002, 415 (24), 429-433.

14. Souman, J.L.; Giordano, P.R.; Frissen, I.; De Luca, A.; Ernst, M.O. Making virtual walking real: Perceptual evaluation of a new treadmill control algorithm. ACM Trans. Appl. Percept. 2010, 7 (2), 1-14

15. Iwata, H. Walking about virtual environments on an infinite floor. In Proceedings of IEEE Virtual Reality Conference, Houston, TX, Mar 13-17, 1999; IEEE Computer Society: Washington, DC; 286-293.

16. Razzaque, S.; Kohn, Z.; Whitton, M.C. Redirected walking, In Proceedings of Eurographics Conference 2001, Manchester, UK, Sept 2011; 289-294.

17. Peck, T.C.; Fuchs, H.; Whitton, M.C. An evaluation of navigational ability comparing Redirected Free Exploration with Distractors to Walking-in-Place and joystick locomotion interfaces. In Proceedings of IEEE Virtual Reality Conference (VR), Mar 19-23, 2011; 55-62. 